is about ten times as rapid as the corresponding decrease. In general, it may be stated that the length of periods and the form of light curves are similar to those of many of the variables in Messier 5, and in 0 Centauri (Astrophysical

Harvard College Observatory, I900 June 18.
Journal, Vol. $X, 255$ ). It will be noted that the periods of these three stars in Messier 3 are about one half a day. Several other variables in this cluster appear to have approximately the same period.

Edward C. Pickering.

Osservazioni del pianeta (433) Eros.

\begin{tabular}{|c|c|c|c|c|c|c|c|c|c|c|c|}
\hline 1900 & T.m.Roma & $\Delta x$ & $\Delta \delta$ & Cf. & Gr. & $\alpha$ app. & $\log p \cdot \Delta$ & $\delta$ app. & $\log p .4$ & Red. ad l. app. & $*$ \\
\hline Lugl. I 7 & $14^{\mathrm{h}} 28^{\mathrm{m}} 6^{\mathrm{s}}$ & - $0^{\mathrm{m}} 7^{\mathrm{s}} .69:$ & $\begin{array}{lr}-3^{\prime} & \text { r. } 5 \\
-2 & 37.8\end{array}$ & $\ddot{6}$ & 12.2 & $1^{\mathrm{h}} 14^{\mathrm{m}} 16^{\mathrm{s}} .22:$ & $\begin{array}{c}9.5^{2} 3 n \\
0.650 n\end{array}$ & $\begin{array}{l}+18^{\circ} 1^{\prime} 26^{\prime \prime} 5 \\
+10\end{array}$ & $\begin{array}{l}0.621 \\
0.602\end{array}$ & $\begin{array}{l}+3^{5.02}+ \\
+3.15+\end{array}$ & 1 \\
\hline
\end{tabular}

Posizioni medie delle stelle di confronto.

\begin{tabular}{|c|c|c|c|}
\hline$*$ & $\alpha 1900.0$ & $\delta$ I 900.0 & Autorità \\
\hline I & $\begin{array}{r}1^{\mathrm{h}} 14^{\mathrm{m}} 20.89 \\
\mathrm{I} \quad 838.65\end{array}$ & $+18^{\circ} 4^{\prime} 15^{\prime \prime}: 4$ & AG. Berlin A. 375 \\
\hline
\end{tabular}

Nota.

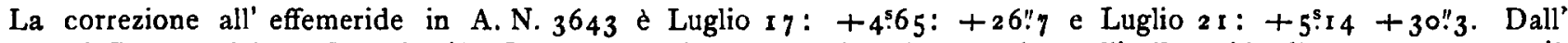
osservazione di Denver del 27 Maggio (A. N. 3642,3647 ), la quale dava la correzione all' effemeride di $+4.14+33^{\prime \prime} \circ{ }^{*}$ ) dovevasi avere al 17 Luglio $+7^{\mathrm{s}} \cdot 53+44^{\prime \prime} \mathrm{I}$ e al $2 \mathrm{x}$ Luglio $+7^{\mathrm{s}} \cdot 97+44^{\prime \prime 6}$ (A. N. $3^{6} 50$ ). In realtà la correzione all" effemeride è più piccola, e pero le correzioni proposte in A. N. 3650 dovranno attualmente moltiplicarsi per $2 / 3$ circa fino a un conteggio ulteriore basato sopra le attuali osservazioni, che saranno sicure.

Roma, I 900 Luglio 23.

E. Millosevich.

*) Wenn man annehmen dürfte, dass die in A. N. 3642 und 3647 mitgetheilten Beobachtungszeiten schon die Verminderung durch die Aberrationszeit enthalten, so würde man für Mai 27 als Correction der Ephemeride +3500 +22." r erhalten, also einen Werth,. der demjenigen, welchen die gegenwärtigen Beobachtungen veriangen, genau entspricht. $K r$.

\title{
Beobachtungen des Planeten (433) Eros
}

am 18-zöll. Refractor der Kais. Universitätssternwarte zu Strassburg von Dr. $H$. Kobold.

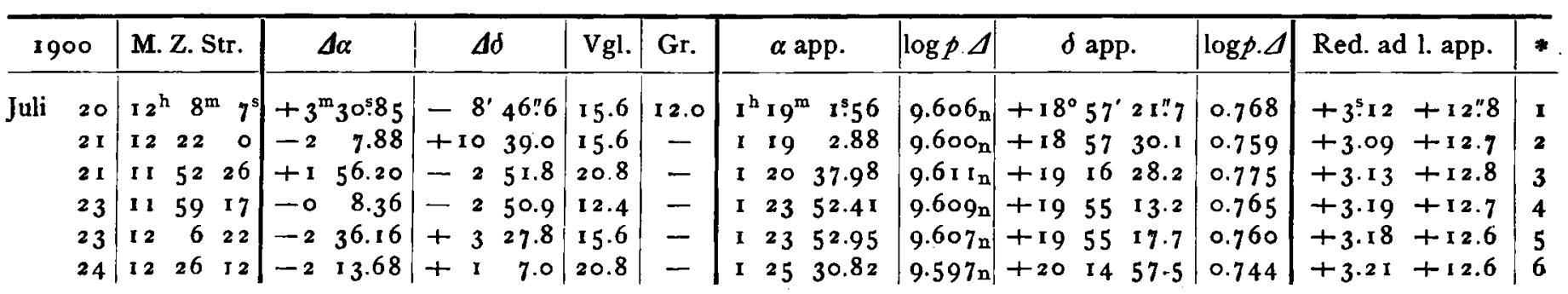

Juli 2 I. Planet meist kaum zu erkennen wegen Dunst und Erhellung des Himmels durch Blitze.

Mittlere Oerter der Vergleichsterne.

\begin{tabular}{|c|c|c|c|}
\hline * & $\alpha 1900.0$ & $\delta 1900.0$ & Autorität \\
\hline I & $1^{\mathrm{h}} 15^{\mathrm{m}} 27^{\mathrm{s}} \cdot 59$ & $+19^{\circ} 5^{\prime} 55^{\prime \prime} .5$ & AG. Berlin A. $38 \mathrm{I}$ \\
\hline 2 & $121 \quad 7.67$ & $+184^{6} 3^{8.4}$ & AG. Berlin A. $4{ }^{1} 3$ \\
\hline 3 & $1 \quad 1838.65$ & +19197.2 & AG. Berlin A. 398 \\
\hline
\end{tabular}

\begin{tabular}{|c|c|c|c|}
\hline * & $\alpha \mathrm{I} 900.0$ & $\delta \times 900.0$ & Autorität \\
\hline 4 & $1^{\mathrm{h}} 23^{\mathrm{m}} 57^{\mathrm{s}} \cdot 5^{8}$ & $+19^{\circ} 57^{\prime} 51^{\prime \prime} 4$ & AG. Berlin A. 422 \\
\hline 5 & $1 \quad 26 \quad 25.93$ & +195137.3 & AG. Berlin A. $43^{6}$ \\
\hline 6 & 12741.29 & +201337.9 & AG. Berlin A. 447 \\
\hline
\end{tabular}

Vergleichung der Beobachtungen mit der Ephemeride in A. N. $3^{643}$ (B - R).

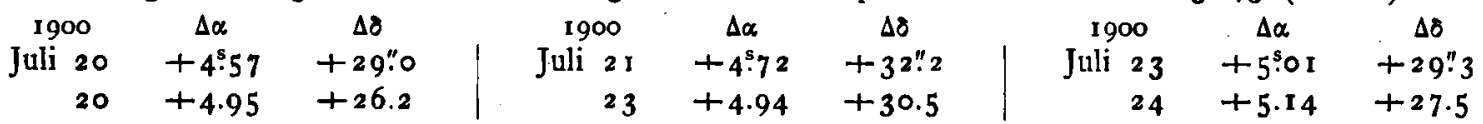

Strassburg, 1900 Juli 25 .

H. Kobold. 